\title{
A ENFERMEIRA NO PROCESSO DE DESCENTRALIZAÇÃO DO SISTEMA DE SAÚDE
}

Maria Aparecida Santa Fé Borges* Maria Ângela Alves do Nascimento**

\section{Resumo}

Estudo sobre o processo de descentralização do sistema de saúde nas décadas de 80 e 90 no município de Itabuna-BA; com o objetivo de descrever o processo de descentralização da saúde no município, identificando a inserção/ participação da enfermeira nesse processo. Estudo descritivo, qualitativo de caráter exploratório, que utilizou para coleta de dados a entrevista semi-estruturada, e a análise documental. Os resultados apontam que a inserção da enfermeira no processo da descentralização seguiu-se de acordo com as mudanças transcorridas a cada gestão municipal, onde ela atuou mais efetivamente conforme foi o cenário estabelecido e sofreu influência das diversas conjunturas conformadas pelas políticas implantadas ou implementadas em cada contexto da gestão do Sistema de saúde municipal.

Descritores: SUS; descentralização; municipalização; enfermeira

\begin{abstract}
Study about the decentralization process of the health system in the '80s and '90s in the city of Itabuna-BA. It is aimed at describing the city's health decentralization process, identifying the nurse's insertion/participation in this process. Descriptive/qualitative study of exploratory nature that utilized both the semistructured interview and documental analysis for data collection. The results show that the nurse's insertion in the decentralization process took place according to the changes that occurred at every municipal management, where the nurse acted more effectively as the set of circumstances was established and was influenced by the several conjunctures formed by the implemented policies or implemented in each context of the management of the
\end{abstract} municipal health system.

Descriptors: SUS; decentralization; municipalization; nurse

Title: The nurse in the decentralization process of the health system

\section{Resumen}

Estudio sobre el proceso de descentralización del sistema de salud en las décadas de 80 y 90 en el municipio de Itabuna-BA; con el objetivo de describir el proceso de descentralización de la salud en el municipio, identificando la inserción/participación de la enfermera en este proceso. Estudio descriptivo cualitativo de carácter exploratorio, que utilizó para la coleta de datos la entrevista semiestructurada, y el análisis documental. Los resultados apuntan que la inserción de la enfermera en el proceso de descentralización se siguió de acuerdo con los cambios transcurridos en cada gestión municipal, donde actuó más efectivamente, conforme fue el escenario establecido y sufrió influencia de las diversas coyunturas conformadas por las políticas implantadas o implementadas en cada contexto de gestión del Sistema de salud municipal.

Descriptores: SUS; descentralización; municipalización; enfermera

Título: La enfermera en el proceso de descentralización del sistema de salud

\section{O Sistema Único de Saúde e a inserção da enfermeira}

Os ideais propostos pela reforma sanitária trazem uma ampliação do conceito de saúde e a democratização do setor saúde, reordenamento do sistema de saúde, mudanças do modelo de assistência e das práticas sanitárias ${ }^{(1-4)}$.

Estas mudanças perpassam pela construção e consolidação do Sistema Único de Saúde - o SUS, em seus princípios e diretrizes, de universalidade, integralidade, equidade, descentralização e participação popular, que demandam novos perfis de profissionais para a organização e prática do novo modelo assistencial na perspectiva da integralidade da assistência.

Portanto, o Brasil vem vivenciando ao longo da última década mudanças operadas na política de saúde, no que tange a construção e consolidação do sistema Único de Saúde-SUS, tendo como base a diretriz da descentralização estabelecida a partir da reforma sanitária, especialmente no processo de municipalização, onde o município passa a ser o condutor responsável por sua política de saúde. Descentralização das ações, do planejamento, dos recursos financeiros e principalmente do poder político.

No entanto, o processo de municipalização não vem acontecendo de forma homogênea em sua implantação nos municípios em decorrência dos entraves político, financeiro e administrativo.

Nessa perspectiva, a ABEn ${ }^{(5)}$ discute em forma de oficinas em vários estados da federação o processo de municipalização e a prática da enfermagem no sentido de compreender e direcionar a prática da enfermagem nas transformações das políticas de saúde e a reformulação do sistema de saúde.

A participação da enfermagem no SUS tem sido objeto de estudo de pesquisadores, que analisam a contribuição da enfermeira no espaço do SUS local através do processo de municipalização. Esses estudos corroboram na importância da participação da enfermeira em diversos estados e Municípios brasileiros na construção do SUS, em suas ações administrativas, assistenciais e educativas, além de suas práticas políticas ainda incipientes, frente às transformações ocorridas, voltadas para a atenção a saúde da população nos serviços de saúde, executando, supervisionando e coordenando as ações de atendimento à população, bem como aponta as dificuldades na construção do novo modelo de atenção a saúde ${ }^{(6-10)}$.

Assim, a municipalização possibilita a ampliação do campo de atuação da enfermeira, favorecendo a participação da sociedade na gestão desse processo já garantido em lei, mesmo enfrentando barreiras, desafios, num movimento histórico e dinâmico, muitas vezes envolto num contexto desfavorável(6-10)

Tal realidade contribuiu com nossa motivação em conhecer como vem se configurando a descentralização da saúde no município de Itabuna-Ba, com a participação/inserção da enfermeira nesse processo.

Neste sentido, buscamos discutir o processo de descentralização da saúde em um dado município baiano, na

\footnotetext{
* Enfermeira. Professora Assistente do Departamento de Ciências da saúde da Universidade Estadual de Santa Cruz UESC) -Ilhéus-BA.Diretora do Departamento da Atenção Básica do Município de Itabuna-BA. **Enfermeira. Orientadora.Professora titular do Departamento de Saúde da Universidade Estadual de Feira de Santana (UEFS)-BA.Doutora em Saúde Coletiva pela Universidade de Ribeirão Preto.Pesquisadora do Núcleo de Pesquisa Integrado em Saúde (NUPISC) do Departamento de Saúde da UEFS.
}

E-mail do autor: nupisc@bol.com.br 
tentativa de fazer uma aproximação da evolução da diretriz que recomenda um conjunto de responsabilidades, de recursos financeiros e de poder para o município dentro da política no Estado nas duas últimas décadas.

No nosso entender a importância desse estudo reside em evidenciar o período histórico pelo qual passou o município de Itabuna/BA. no processo de construção do SUS. Trata-se de um período não explorado anteriormente, que descreve a participação da enfermeira nesse processo. Partindo desse entendimento, estabelecemos como objetivo descrever o processo de descentralização da saúde no município de Itabuna/ BA. nas décadas de 80 e 90, identificando a inserção/ participação da enfermeira nesse processo.

\section{Metodologia}

Trata-se de um estudo descritivo, com abordagem qualitativa de caráter exploratório. Tomamos como campo de estudo o município de Itabuna, delimitando a rede básica e o serviço público de saúde como abrangência da pesquisa. O município de Itabuna faz parte da micro-região geográfica sulbaiana, com uma população estimada de 181.752 habitantes. É considerado de médio porte, um pólo de desenvolvimento regional.

Em relação à saúde, possui uma rede composta por 19 Unidades Básicas de Saúde-UBS, (centros e postos de saúde), funcionando nos turnos: matutino e vespertino. Dessas UBS, seis contam com atuação de uma enfermeira em cada turno; 11 funcionam com apenas uma enfermeira por turno. Dessas UBS, duas funcionam na zona rural, com atuação da enfermeira em dias programados.

Os dados foram coletados através da entrevista semiestruturada, dos documentos técnicos (regimentos, decretos lei, lei orgânica) e oficiais (relatórios, planos de saúde, livros, ata) relativos às políticas de saúde implementadas no município nas décadas de 80 e 90, que evidenciavam a inserção da enfermeira no sistema de saúde e sua rede de unidades básicas, relacionando-os às falas das entrevistadas, inclusive o confronto com os seus discursos.

Os sujeitos da pesquisa foram 16 enfermeiras, agrupadas em: enfermeiras informantes-chave (aquelas que participaram e/ou participam do processo da descentralização em Itabuna/BA - grupo I); enfermeiras que atuam na coordenação dos serviços de saúde - grupo II) e enfermeiras que atuam nas UBS - grupo III). Neste artigo são apresentados os dados obtidos a partir dos 03 sujeitos do grupo I, enfermeiras informantes-chave.

Em relação aos aspectos éticos, foi solicitada a permissão para as entrevistas através do termo de consentimento individual. Procuramos resguardar a fidedignidade das falas, nos discursos das entrevistadas, obedecendo à forma de falar de cada uma. Os depoimentos são identificados, obedecendo ao critério de ordem crescente de cada entrevista realizada, por exemplo, entrevistada 3, lê-se Ent. 3, acompanhada do grupo do qual pertença (grupo I, grupo II, grupo III) ou seja: (Ent. 3. grupo I).

\section{A trajetória da descentralização}

Em Itabuna o processo de descentralização da rede de saúde foi iniciado com o convênio n. ${ }^{\circ}$ 02/85 através da estratégia das Ações Integradas de Saúde - AIS ${ }^{(11)}$, em 1984, priorizando nesse primeiro momento, a unificação das ações de saúde dos três níveis de governo: federal, estadual e municipal. A primeira ação integrada pelo município foi a implantação do Sistema Integrado de Vigilância Epidemiológica - SIVE, que passou a ter na coordenação uma enfermeira do Serviço Especial de Saúde Pública - SESP, uma enfermeira da Secretaria de Saúde do município e uma enfermeira da Sétima Diretoria Regional de Saúde - 7. ${ }^{\text {a DIRES }}$

Implementar as AIS significava a integração dos serviços e o entendimento da descentralização como repasse de recursos financeiros, mediante convênio para a manutenção da prestação de serviços nas unidades da rede municipal apesar dos

[...] técnicos que compunham a comissão local interinstitucional de saúde entenderem a perspectiva da reorganização da rede de serviços de saúde do município, de modo a oferecer atenção integral à população segundo níveis de complexidade crescente utilizando forma planejada e racional a capacidade máxima dos recursos disponíveis, melhorando a cobertura desses serviços. (Ent. 1, grupo I).

[...] o município de Itabuna já estava avançando nesse processo, inclusive com a criação da Comissão Interinstitucional municipal de saúde -CIMS, a CRIS ${ }^{a}-$ integrada pelo Diretor do SESP, Diretor do INAMPS, o diretor da 7. a DIRES e o Secretário Municipal de Saúde. Concomitantemente foi formada a comissão local Interinstitucional de saúde- CLIS ${ }^{\mathrm{b}}$ - composta por enfermeiras da Secretaria Municipal de Saúde, 7. ${ }^{a}$ DIRES e SESP. (Ent. 1, grupo I).

Esse momento foi muito importante para as três únicas enfermeiras do município, pois, além de suas ações de supervisão e organização dos serviços de saúde elas participavam ativamente do processo de mudança na saúde que estava ocorrendo em Itabuna a partir da integração da vigilância Epidemiológica aos três níveis de governo.

[...] O sistema integrado de vigilância epidemiológica possibilitou uma mudança no modelo de assistência à saúde porque integrava as técnicas dos três níveis de governo (SESP, DIRES e S.M.S), onde com o planejamento e supervisão foi possível estabelecer cobertura vacinal, coordenação de imunização com funcionamento da rede de frio e treinamento para os servidores que atuavam na rede básica de saúde. Houve um fortalecimento qualitativo (Ent. 2, grupo I).

Este depoimento demonstra o processo importante que passava o município em relação à organização do sistema de saúde pois,

[...] inclusive nós naquela época (1984), me lembro que nós recebíamos vários técnicos do Paraná, Brasília para ouvirem e conhecerem esse sistema integrado de vigilância. Diríamos que a seriedade era tão grande que técnicos do ministério da saúde se deslocaram para fazer parte desse processo das AIS que estava acontecendo em Itabuna (Ent.1, grupo I).

No período de 1983 a 1988 existia na Secretaria de Saúde apenas três enfermeira participando da descentralização da saúde no município. Nessa época das AIS a ação da enfermeira no planejamento e na supervisão talvez facilitasse o desenvolvimento desse processo

[...] Eu me lembro que naquela época das AIS quando nós reuníamos toda a equipe de saúde para reunião com o prefeito e tudo, os médicos começaram a dizer que as enfermeiras eram o staff da prefeitura, porque eles achavam que a gente tinha uma posição destacada (Ent.1, grupo I).

A enfermeira nesse momento se insere no sistema e no serviço saúde, onde participam do processo de construção da descentralização após a realização de seminários e reuniões técnicas na Secretaria de Saúde e na rede através da organização dos serviços voltados para a atenção à criança e à mulher em relação à imunização e pré-natal apesar de que, nessa época, a consulta da enfermagem não era considerada

\footnotetext{
a Comissão Regional Interinstitucional de Saúde - órgão gestor colegiado previsto pelas AIS para coordenar as ações de saúde em nível regional.

b Comissão Local Interinstitucional de saúde - órgão gestor técnico colegiado previsto pelas AIS para coordenar as ações de saúde em nível local.
} 
como uma atividade institucionalizada. Segundo as informanteschave esse grupo entendia o processo que estava ocorrendo no Brasil, na Bahia e em Itabuna (Ent. 1; grupo I).

[...] a gente começou organizando os serviços nas ações, primeiro com vacinação, notificação, investigação epidemiológica, depois a gente passou para outros serviços. Também criamos a comissão técnica integrada para supervisão dos serviços em todos os postos da prefeitura. (Ent. 3, grupo I).

Percebemos nesse período o avanço do município, pois enquanto no país estava ocorrendo o processo das AIS em Itabuna era aprovada na Câmara Municipal, o Código de Postura através da Lei 1331 de 08 de janeiro de $1985^{(12)}$ que traduz nos aspectos legais a política administrativa de responsabilidade do município em matéria de proteção à saúde, da ordem pública e proteção ao verde, prevenção de incêndio e combate ao fogo e dos estabelecimentos comerciais e industriais. Estatuindose as relações entre o poder público local e os munícipes, ao assumir zelo pela promoção, proteção da saúde e pelo bemestar público da coletividade, avançando nos aspectos legais a responsabilidade municipal nas questões de saúde, ou seja,cinco anos antes da elaboração da Lei Orgânica do Município que foi promulgada em 05 de abril de 1990(13).

Ainda, nesta gestão municipal, Itabuna celebra o termo de adesão ao aditivo financeiro para operacionalização do Sistema Unificado e Descentralizado de Saúde-SUDS ${ }^{(14)}$, com a transferência dos recursos financeiros condicionada à construção da CIMS (já constituída no município) e instituição do Conselho Municipal de Saúde, e o plano municipal aprovado pelo Conselho municipal de saúde.

Com a eleição municipal de 1988, ocorrem mudanças na gestão municipal.

[...] Em 1989, com mudança da gestão através das eleições municipais, assume o município o prefeito Fernando Gomes, havendo também mudanças na direção do SESP e 7. ${ }^{a}$ DIRES, ocorrendo uma desacumulação do processo de descentralização da saúde em Itabuna (Ent. 2, grupo I).

Neste contexto a Secretaria de Saúde do Estado transfere para o município a gestão de todos os sete postos de saúde sob seu comando, assim como os termos específicos de cessão de uso, bens e equipamentos, também à sessão de pessoal pertencentes ao Estado.

O credenciamento do município de Itabuna desde as AIS ao SUDS, possibilitou a expansão da rede básica, ocorrendo nesse período, uma transformação na estrutura da rede de assistência (efetivada na gestão de 1983 a 1988). O empenho por esse aumento estava de acordo com a estratégia das ações integradas em saúde de descentralizar, regionalizar e integrar esses serviços de saúde. Em relação à rede básica, embora tenha crescido, houve uma tendência na ampliação da estrutura física das unidades e no aumento da capacidade e especialização no atendimento à saúde ${ }^{(15)}$. Inclusive o município se estruturou com significativa rede de postos de saúde situados nos bairros periféricos da cidade e na zona rural.

A rede básica foi estruturada com um conjunto de unidades de diferentes níveis de complexidade de atenção das diversas instituições prestadoras de serviços. Apresenta área geograficamente delimitada de intervenção definida com uma população adscrita a essa unidade ${ }^{(15)}$. Desse modo, o sistema local foi organizado em quatro módulos, onde em cada um deles foi instituída uma unidade básica de saúde de referência por seu maior nível de complexidade no atendimento. No entanto o sistema de referência e contra-referência preconizado nesta forma de organização, não funciona na prática devido à desarticulação entre a rede básica, os laboratórios e os hospitais. Ou seja, a desarticulação entre a atenção básica, a média e alta complexidade.

Em Itabuna, no período de 1989 a 1992 a rede básica aumentou em sua estrutura e capacidade instaladas devido à transferência de unidades de saúde e equipamentos de outro nível de governo, bem como houve um incremento de recursos humanos transferidos do Estado para o município.

Em referência aos recursos humanos, começa a ocorrer expansão no município a partir do final da década de 80 e início da década de 90 devido à necessidade de reorganização do sistema de saúde assim como pela transferência de servidores estaduais para a gestão municipal, que começa a assumir uma "problemática" do processo de descentralização, passando o sistema local a contar em suas instituições servidores com níveis e cargos salariais diferenciados, acarretando dificuldades de manutenção de pessoal na prestação de serviços.

Entendemos que esta situação continua gerando conflitos ocasionados pela resistência às mudanças. Não há registro de discussão acerca da descentralização com estes servidores, levando-os assim a insatisfação devido à rede de serviços ser ainda inadequada para a municipalização(16).

Particularmente em 1992 houve um incremento de recursos humanos na rede básica devido à realização do concurso público municipal para o setor saúde. Em relação à enfermagem, foram admitidas sete enfermeiras na rede básica, com função de supervisão e administração de recursos materiais das unidades e de pessoal de enfermagem ${ }^{(17)}$. Neste mesmo ano foi implantado o Programa de Agentes comunitários de saúde - PACS.

Em 1993 ocorre nova eleição municipal, mudando assim o gestor municipal. É eleito um prefeito de frente progressista. Assume a Secretaria de Saúde um médico sanitarista como secretário de saúde e uma enfermeira como assessora técnica. Retoma novamente o processo de discussão da municipalização com a participação dos segmentos da sociedade e a (re) estruturação do Conselho Municipal de Saúde, através da definição das entidades representativas para este Conselho. Vale ressaltar que a Associação Brasileira de Enfermagem - Regional Itabuna- (ABEn) teve assento garantido no CMS, conforme observamos nesse depoimento

\begin{abstract}
[...] A gente volta à cena porque, como nós tínhamos criado a ABEn, a gente brigou para que a ABEN tivesse um assento no Conselho. Então nós conseguimos ter esse assento no Conselho. E é quando a gente volta nesse processo a discutir Itabuna, participando no Conselho dessas discussões também (Ent. 2, grupo I).
\end{abstract}

Este depoimento demonstra a vontade da ABEn, sociedade civil organizada representante da Enfermagem em participar do processo de (re) organização do Sistema de Saúde. entretanto a reconstrução foi difícil. Embora o gestor municipal desejasse um avanço na saúde, o seu entendimento sobre saúde ainda é limitado, pois ele desconhece o processo de descentralização não entendendo a necessidade de maior autonomia para a Secretaria de Saúde desencadear o processo de municipalização, pois,

[...] o gestor não tinha essa clareza do que era municipalização e do que era descentralização. Quando eu falo dessa descentralização, seria a descentralização em termos de recursos também, essa autonomia da própria secretaria (Ent. 1, grupo I).

Percebemos que durante o processo de descentralização a equipe técnica precisou de tempo para elaborar a proposta de reorganização do sistema de saúde local, começando justamente pela participação da população através do controle social. Intitui-se o processo de discussão com os servidores de saúde municipal no sentido de implantar a nova política de saúde adotada pela nova gestão, tendo a participação então, desse corpo técnico da SMS, de uma enfermeira como assessora.

Em julho de 1994, o município institui o Fundo Municipal de Saúde ${ }^{(18)}$ e a lei, que dispõe sobre a participação da comunidade na gestão do SUS ${ }^{(19)}$, o Conselho Municipal de 
Saúde e as Comissões Locais de Saúde como órgãos colegiados permanentes de caráter consultivo e deliberativo integrante da estrutura do sistema municipal de saúde, obedecendo as leis orgânicas da saúde. Neste mesmo ano, o município assume a gestão do sistema de saúde na gestão incipiente ${ }^{(20) c}$.

Na forma de gestão da Norma Operacional Básica de 1993 (NOB-SUS 01/93), o sistema de saúde do município começa a ser organizado como prestador de serviços e passa a receber os seus proventos através de serviços produzidos. Assim, a gestão da assistência ambulatorial também se dá em nível hospitalar e não só na rede básica. As unidades públicas e contratadas com respectivos serviços, recursos humanos e produções são acompanhadas pelo menos para o financiamento através do SIA e SIH/SUS.

Com a intenção de implementar a municipalização, o município começa a reestruturar a rede básica através da contratação de profissionais de saúde multidisciplinares para que atendessem as necessidades de saúde da população.

Nesse intuito foram contratadas enfermeiras para todas as unidades de saúde, perfazendo um total de 33 enfermeiras, atuando na gerência informal ${ }^{\mathrm{d}}$ (grifo nosso), com o apoio do nível central, assumindo o planejamento e direção das ações de saúde, a supervisão dos procedimentos de enfermagem, o controle de higiene e dos recursos materiais, além da assistência ao pré-natal de baixo risco e o acompanhamento do crescimento e desenvolvimento da criança, as atividades de educação em saúde e as visitas domiciliares em conjunto com os agentes comunitários de saúde, através de ações programáticas, associadas à demanda espontânea(21).

Concretamente a enfermagem neste período apresenta uma importância quali-quantitativa como força de trabalho no setor saúde, contribuindo com a reorganização dos serviços na construção do SUS local ${ }^{(9,22)}$.

Entendemos que no período de 1993 a 1996, houve uma retomada da discussão sobre a municipalização da saúde, tendo uma das autoras deste estudo participado do processo, inclusive como trabalhadora de saúde na rede básica de saúde. A enfermeira volta a se inserir de uma forma mais ativa nesse processo, com maior autonomia, contudo continua desenvolvendo na maioria das vezes, atividades na área da assistência.

Durante o processo da municipalização da saúde em Itabuna, a enfermeira enfrentou dificuldades na (re) organização dos serviços. Lentamente ela foi se inserindo como uma técnica da atenção à saúde e como um agente que veio também para construir a municipalização da saúde. Na realidade passou a assumir diversas atividades surgidas a partir da descentralização das ações e serviços de saúde para atender a demanda reprimida.

Ainda assim, a participação da enfermeira na municipalização contribui com a melhora do atendimento prestado a população numa perspectiva da integralidade da assistência e amplia a sua participação e autonomia ${ }^{(7,9,10,23)}$, conforme depoimento a seguir,

[...] O enfermeiro então era quem fazia a consulta, nós tivemos a participação dos enfermeiros nos conselhos locais de saúde. Então foi muito importante a enfermeira nessa parte do procedimento, de oferecer ações mais integrais para a população. Então a enfermeira assume esse papel agora em termo de avaliação mesmo de qualidade de serviço entendeu? (Ent. 1, grupo I).

\footnotetext{
c Nesta forma de gestão o município é habilitado após criar o Fundo Municipal de Saúde e o Conselho Municipal de Saúde passando a receber os recursos financeiros mediante prestação de serviços ao SUS e um adicional a título de estímulo à municipalização. ${ }^{21}$

d Gerência Informal - porque não existe este cargo na estrutura da Secretaria Municipal de Saúde e o profissional não recebe proventos por assumir tal função.
}

Outro fato importante foi a I Conferência Municipal de Saúde ${ }^{(24)}$, realizada em agosto de 1995 com discussões sobre a participação popular nas políticas de saúde em Itabuna, quando tivemos a oportunidade de participar como delegada representante dos prestadores de serviço (SMS) das comissões locais de saúde.

Essa Conferência foi um marco na história da saúde para o município, porque possibilitou a discussão do modelo de saúde que Itabuna iria adotar, o financiamento das ações de saúde e o controle social, através do Conselho Municipal e dos Conselhos Locais sobre estas ações ${ }^{(25)}$.

Em 1996, ocorre nova eleição para prefeito e Itabuna mais uma vez muda seu gestor. Contudo, o processo de municipalização da saúde continua, até por exigência das normas que o Ministério da Saúde edita, no sentido de manter a descentralização e a efetivação do SUS.

Nesse período, Itabuna assume a gestão plena da atenção básica ${ }^{(17) e}$, reestrutura e regulamenta o Conselho Municipal de Saúde, revogando as disposições da lei municipal n. ${ }^{\circ} 1667$ de julho de 1994(19), alterando neste sentido a composição das instituições participantes do $\mathrm{CMS}^{(26)}$.

É criada a estrutura da Secretaria de Saúde ${ }^{(27)}$, que tem por finalidade a formulação, coordenação e execução da política de saúde do município mediante ações próprias e articuladas com outros órgãos públicos federais, estaduais, municipais e entidades particulares, objetivando o acesso universal e igualitário nos serviços de saúde.

Todavia, apesar do sistema de saúde do município vir se reestruturando, durante este período não houve expansão da estrutura física da rede básica que se mantém a mesma desde a década de 80 , mesmo com a inserção de diversos profissionais de saúde de nível superior no gerenciamento desses serviços.

Atualmente Itabuna dispõe de uma rede de serviços de média e alta complexidade, oferecendo serviços na área de urgência/emergência, traumo-ortopédico, hematologia, radioterapia, oftalmologia, medicina nuclear, terapia renal substitutiva, fisioterapia, anatomia patológica, citopatologia, radiodiagnóstico, endoscopia, tomografia computadorizada, patologia clínica dentre outros, num quantitativo que lhe garante o conceito de pólo regional na área hospitalar, fortalecendo assim o crescimento do sistema secundário e terciário na atenção à saúde.

No entanto, o mesmo não ocorre com a rede do sistema público, pois não houve ampliação das unidades básicas de saúde, as quais são enfraquecidas mediante o fortalecimento do atendimento individual curativista, direcionado à queixa/ conduta, através do projeto saúde em sua casa ${ }^{(17)}$. Privilegiando o atendimento em ruas e praças dos bairros periféricos através de carros montados para o atendimento clínico, ginecológico, oftalmológico e odontológico, descaracterizando o SUS em seus princípios de universalização, integralidade e equidade, comprometendo as ações de saúde desta população.

\section{Considerações finais}

Itabuna nessas décadas vivenciou um processo de transformação em quatro momentos distintos de acordo com as mudanças de gestão, com características de um desenvolvimento desigual em seu processo de descentralização. Sua política institucional oscilou nas diversas conjunturas expressada através das estratégias implantadas ou implementada em seus períodos determinados. Assim, os

\footnotetext{
Nesta forma de gestão o município adquire autonomia financeira para gerir toda rede básica, como também ações de vigilância epidemiológica e sanitária.O mecanismo de financiamento se dá através do PAB (Piso Assistencial Básico), que corresponde aos recursos através de crédito populacional para o financiamento das ações de saúde.o valor a ser transferido aos municípios é de acordo com o número de habitantes ${ }^{25}$.
} 
avanços ou retrocessos aconteceram conforme foi à compreensão por parte dos gestores municipais do processo de descentralização/municipalização, favorecendo ou dificultando dessa maneira a atuação dos trabalhadores de saúde, dentre eles a enfermeira.

A enfermeira se inseriu na realidade em quase todos esses momentos acima referidos e a depender do cenário estabelecido ela participou mais efetivamente, sofrendo a influência das diversas conjunturas conformadas pelas políticas implantadas em cada contexto.

Desta maneira, a inserção da enfermeira nesse processo foi seguida de acordo com as mudanças transcorridas em cada momento vivenciado pela gestão municipal.

Entendemos que os resultados apresentados neste estudo em relação à participação da enfermeira não podem estar desvinculados a um contexto que é histórico e social, estando em constantes transformações, sejam elas positivas ou negativas, a depender do olhar de cada ator social. E por isso, imprimindo uma participação dessa trabalhadora (enfermeira), muitas vezes com limitação em decorrência das políticas de saúde ora vigente que não se compromete com a saúde da população, e assim mantém um sistema de saúde debilitado, fragmentado, porque persiste num modelo de assistência, centrado na doença, no indivíduo e no tecnicismo que não atende na maioria das vezes as necessidades de saúde da população.

No entanto, ressaltamos a importância da participação da enfermeira na efetivação do Sistema único de Saúde mediante o desenvolvimento de sua prática sanitária e sua participação política enquanto sujeito social desse sistema.

\section{Referências}

1. Arouca AS. Reforma sanitária. Rio de janeiro: Fiocruz;1988.

2. Campos G W S. A saúde pública e a defesa da vida. São Paulo: Hucitec;1992.

3. Campos G W S. A reforma da reforma: repensando a saúde. $2^{\mathrm{a}}$ ed. São Paulo: Hucitec, 1997.

4. Mendes E V. Distrito sanitário: o processo social de mudanças das práticas sanitárias do sistema Único de Saúde. São Paulo: Hucitec;1995.

5. Associação Brasileira de Enfermagem (ABEn). Relatório da oficina de trabalho: o processo de municipalização e a prática de enfermagem. Brasília (DF): ABEn;1992.

6. Melo C, Araújo MAGSA. A nova prática de enfermagem no sistema único de saúde. In: Descentralização em saúde e a prática de enfermagem. Cadernos da ABEn/Nacional. Brasília (DF):ABEn; 1992.

7. Antunes MJM. O enfermeiro e a integralidade da assistência de enfermagem na rede básica do sistema único de saúde [dissertação de Mestrado em Enfermagem]. Belo Horizonte (MG): Escola de Enfermagem, Universidade Federal de Minas Gerais;1996.

8. Almeida MCP, Rocha SM. O trabalho de enfermagem. São Paulo: Cortez;1997.

9. Cheade MFM. Caracterização das atividades dos enfermeiros nas secretarias municipais de saúde do Estado de Mato Grosso do Sul [dissertação de Mestrado em Enfermagem]. São Paulo: Escola de Enfermagem, Universidade de São Paulo;2000.
10. Shimizu HE, Santos ER. Percepções de gerentes sobre a assistência à saúde coletiva. Revista Brasileira de Enfermagem, Brasília (DF) 2002 mar/abr;55(2): 157-62.

11. Secretaria de Saúde de Itabuna (BA). Termo de adesão da prefeitura municipal de Itabuna. Convênio AIS 02/85. Visa transferir recursos para as ações integradas de saúde. Itabuna (BA):1985.

12. Itabuna (BA). Lei no 1331 de 08 de janeiro de 1985. Constitui o Código de Postura e dá outras providências. Itabuna (BA): Jornal Oficial;1985.

13. Itabuna (BA). Lei Orgânica Municipal da Saúde. Câmara Municipal dispõe sobre as condições para proteção e recuperação da saúde, organização e funcionamento dos serviços correspondentes e dá outras providências. Itabuna (BA): Câmara Municipal;1990.

14. Secretaria de Saúde da Bahia (BA). Decreto no 94657 de 20 de julho de 1987 firma o termo de adesão ao termo aditivo do convênio SUDS/Ba-87, visando adotar procedimentos adequados à transferência dos recursos necessários à operacionalização do Sistema Unificado e Descentralizado de Saúde para o exercício de 1988. Salvador (BA):SSB;1988.

15. Borges MASF. Avaliação da situação nutricional das crianças de 0 a 5 anos, atendidas na Unidade de Saúde Alberto Teixeira Barreto, residentes no Bairro Nova Califórnia, Itabuna -Ba. Abril/94 a Maio/95 [monografia]. Ilhéus (BA): UESC;1995.

16. Secretaria de Saúde Bahia (BA). Plano estadual de saúde 19961999. Salvador (BA);1995.

17. Secretaria Municipal de Saúde de Itabuna (BA).Relatório de gestão. Itabuna(BA);1997.

18. Itabuna (BA). Lei $n^{\circ} 1668$ de 13 de julho de 1994. Institui o Fundo Municipal de Saúde. Itabuna (BA): Jornal Oficial;1994.

19. Itabuna (BA).Lei $n^{\circ} 1667$ de 13 de julho de 1994 dispõe sobre a participação da comunidade na gestão do SUS. Cria o Conselho Municipal de Saúde e institui as comissões locais de saúde e as conferências municipais de saúde.Itabuna (BA): Jornal Oficial;1994.

20. Secretaria de Saúde da Bahia (BA). Processo n 3009408932889 , em 21 de dezembro de 1994. Aprova a gestão incipiente do município de Itabuna-Ba. Salvador (BA);1994.

21. Ministério da Saúde (BR). Descentralização das ações e serviços de saúde: a ousadia de cumprir e fazer cumprir a lei. Brasília( DF):1993.

22. Sena-Chompré RR, Egry EY. A enfermagem nos projetos uni: contribuição para um novo projeto político para a enfermagem brasileira. São Paulo: Hucitec;1998.

23. Antunes MJM, Egry EY. O programa saúde da família e a reconstrução da atenção básica no SUS: a contribuição da enfermagem brasileira. Revista Brasileira de Enfermagem, Brasília (DF) 2001 jan/mar;54(1):98 $-107$.

24. Conselho Municipal de Saúde de Itabuna (BA). Institui a I conferência Municipal de saúde. Itabuna (BA);1995.

25. Ministério da Saúde (BR). Norma Operacional Básica do SUS - NOB 01/96. Brasília (DF);1996.

26. Itabuna (BA). Lei $n^{\circ} 1749$ de 28 de agosto de 1997 altera os dispositivos da lei municipal n¹667 de 13 de julho de 1994. Altera a estrutura e regulamentação do CMS e dá outras providências. Mantém o sistema de modularização. Itabuna (BA): Jornal Oficial;1997.

27. Itabuna (BA). Decreto 5272 de 02 de agosto de 1997 cria a estrutura administrativa e a estrutura da Secretaria Municipal de Saúde.Itabuna (BA): Jornal Oficial;1997.

Data de Recebimento: 22/09/2003

Data de Aprovação: 22/12/2004 